Tropical Journal of Pharmaceutical Research, December 2007; 6 (4): 815-824

(C) Pharmacotherapy Group Faculty of Pharmacy, University of Benin Benin City, Nigeria.

All rights reserved.

Research Article

Available online at http://www.tjpr.org

\title{
Preparation of Polymeric Micelles for Use as Carriers of Tuberculostatic Drugs
}

\author{
Márcia Silva $^{1^{*}}$, Eiizabeth I. Ferreira ${ }^{2}$, Clarice Q. F. Leite ${ }^{3}$, and Daisy \\ N. Sato ${ }^{4}$, \\ 1 epartamento de Fármacos e Medicamentos, Faculdade de Ciências Farmacêuticas, Unesp, Araraquara - SP, Brazil \\ 2 epartamento de Farmácia, Faculdade de Ciências Farmacêuticas, USP, São Paulo - SP, Brazil \\ ${ }^{3}$ epartamento de Ciências Biológicas, Faculdade de Ciências Farmacêuticas, Unesp, Araraquara - SP, Brazil \\ ${ }^{4}$ nstituto Adolfo Lutz, Ribeirão Preto - SP, Brazil
}

\begin{abstract}
Purpose: This paper focuses on the characterization of polymeric micelle-forming tuberculostatic prodrugs and the antimycobacterial activity of these prodrugs.

Method: By the condensation of hydroxymethylpyrazinamide, isoniazid and rifampin with free carboxyl groups on the copolymer poly(ethyleneglycol)-poly(aspartic acid), micelle-forming carrier-drug conjugates were obtained. These micelles were characterized by dynamic light scattering, to measure the micelle diameter; by acid-base titration, to determine the percentage of carboxylic groups occupied by the tuberculostatic; by Sudan III solubility tests, to estimate the critical micelle concentration (CMC); and visual control and spectrophotometric measurement, to determine the stability of micelles. These micelles were tested in vitro against several Mycobacterium strains.

Results: As expected, the size and distribution of the micelle-forming tuberculostatic prodrugs found to be small $(78.2 \mathrm{~nm}, 84.2 \mathrm{~nm}$ and $98.9 \mathrm{~nm}$ ) while the level of the drug conjugated was high (65.02-85.7\%). Furthermore, the micelles were stable in vitro, exhibiting a low level of CMC and stronger antimycobacterial activity than the original drugs.

Conclusion: The results demonstrate that polymeric micelles can be used as efficient carriers for drugs, which alone, exhibit undesired pharmacokinetics, poor solubility, and low stability. The synthesized micelle-forming tuberculostatic prodrugs opens a perspective of alternative prodrugs that prolong action and decrease the toxicity of the tuberculostatic drugs of choice.
\end{abstract}

Keywords: pyrazinamide, isoniazid, rifampin, tuberculostatic prodrugs, polymer micelles

${ }^{*}$ Corresponding Author: Tel.: 055-16-33016971, Fax: 055-16-33016960; email: silvam@fcfar.unesp.br 


\section{INTRODUCTION}

Despite the great amount of scientific data amassed on tuberculosis (TB), an infection that has afflicted the human race for thousands of years, there is still no victory in sight over the disease, which today represents a severe problem for public health in many parts of the world, especially in the poorer countries. With the constantly growing number of multidrug resistant strains and the spread of AIDS, which destroys patients`immunity, leaving them much more susceptible to mycobacterial infection, the rates of incidence and prevalence of TB have been rising and could threaten the socioeconomic development of affected countries, in view of the fact that both TB and AIDS affect, in the main, people in their most productive years, aged $15-49^{1,2}$.

While it is true that very useful tuberculostatic products exist, both for treatment and prophylaxis, the known toxic side-effects of these drugs, the infection of patients by the $M$. avium complex and their lack of adherence to the course of treatment, are all factors that make the ongoing search for improved drugs absolutely indispensable. As a rule, new drugs are introduced by modifying molecules with known characteristics, often with the help of computer-aided design techniques ${ }^{3}$. Synthetic routes that are designed to begin with a known drug and make planned improvements in some of its properties, particularly in its pharmacokinetics (as is the case in delayedaction prodrugs) are often used ${ }^{3,4,5}$. Prodrugs are chemicals with little or no pharmacological activity, undergoing biotransformation to a therapeutically active metabolite. In the prodrug approach, the physicochemical properties of the parent drug are transiently modified with a promoiety or carrier ${ }^{4}$. The objectives of a prodrug strategy are: to solve problems resulting from poor solubility, insufficient chemical stability or poor organoleptic properties; reduced systemic toxicity; improved oral bioavailability, by improving the oral absorption of the drug and/or by decreasing its presystemic metabolism. Other objectives are to improve absorption, lengthen the duration of action of the drug by slow metabolic release, and finally achieve the organ/tissue-selective delivery of an active agent $^{3,4}$.

Even though great advances have been made in polymeric drugs, the practice of conjugating hydrophobic drug moieties in dense clusters along the polymer chain of a carrier molecule and, not infrequently, the low water solubility of the carrier itself, can easily result in the precipitation of the polymer derivative, and often does $5,6,7,8$. To overcome this problem, a promising technique is to make polymer drugs that take the form of micelles ${ }^{5,6,7,8}$. In line with this approach, micelle-forming polymer derivatives of the initial-phase anti-tuberculous drugs - pyrazinamide, isoniazid and rifampin were synthesized in this study. These derivatives were characterized by determining the critical micelle concentration (CMC) and the mean diameter of the micelles, and then tested for activity against several mycobacterial strains, such as virulent $M$. tuberculosis and M. avium.

\section{Materials and methods \\ Chemicals}

Pyrazinamide (PZA), isoniazid (INH) and rifampin (RMP) were obtained from FURP (Popular Remedy Foundation), $\alpha$-methyl- $\omega$ aminopoly(oxyethylene) $\left(\mathrm{CH}_{3}-\mathrm{PEG}-\mathrm{NH}_{2}, \mathrm{MW}\right.$ $5,000)$ was purchased from Shearwater, Inc., USA, and 1-ethyl-3-(3-dimethylaminopropyl) carbodiimide (EDC) from Aldrich. Other reagents were obtained from a variety of commercial sources.

\section{Synthesis}

The synthetic pathways of the micelle-forming polymer derivatives of PZA, INH and RMP are shown in Scheme 1. The synthetic procedures used have been published in detail elsewhere 9,10 . The products isolated at each step were lyophilized. $\quad \beta$-Benzyl-L-aspartate $\quad[\mathrm{BLA}]^{11}$ compound (3), was produced by allowing aspartic acid and benzyl alcohol to react in sulfuric acid for $24 \mathrm{~h}$, with magnetic stirring, at $13-17^{\circ} \mathrm{C}$, and isolated by adding pyridine and chilling at $-30^{\circ} \mathrm{C}$ overnight, to precipitate the product, which was recrystallized from water by adding pyridine dropwise. Phosgene gas was bubbled through a suspension of BLA in dioxan, 
at $60^{\circ} \mathrm{C}^{12,13}$, to obtain a solution of compound (5), $\quad \beta$-benzyl-L-aspartate $N$-carboxyanhydride (NCA-BLA). Solvent and excess phosgene were removed and the product mixed with $1: 1$ petroleum ether / ethyl acetate; the resulting precipitate, after being filtered, was taken up in $1: 1$ chloroform/butanol mixture and chilled at $30^{\circ} \mathrm{C}$ to isolate solid NCA-BLA. The latter was allowed to react with $\mathrm{CH}_{3}-\mathrm{PEG}-\mathrm{NH}_{2}$ for $24 \mathrm{~h}$ in a stirred solution, under flowing argon, and then sonicated for 30 min to give compound (7), the copolymer poly(ethylene glycol)-poly( $\beta$-benzyl-Laspartate) (PEG-PBLA) $)^{5,6}$ which was precipitated in 1:1 ethanol/water, redissolved in chloroform and reprecipitated in 2-propanol at $40^{\circ} \mathrm{C}$. Next, alkaline hydrolysis of this copolymer at $0^{\circ} \mathrm{C}$, carried out with $0.43 \mathrm{M} \mathrm{NaOH}$ dissolved in 1:2:2 water/propanol/methanol, led to compound (8), poly(ethylene glycol)poly(aspartic acid) (PEG-PASP) ${ }^{5,6}$. After 10min of stirring, the alkali was neutralized with acetic acid and the mixture poured into ethyl ether. The precipitate was filtered, dissolved in water and dialyzed against distilled water for $4 \mathrm{~h}$.

Hydroxymethylpyrazinamide (compound11, $\left.\mathrm{PZACH}_{2} \mathrm{OH}\right)^{10}$ was produced by mixing pyrazinamide, $4 \%(\mathrm{w} / \mathrm{v})$ potassium carbonate solution and $40 \%(\mathrm{v} / \mathrm{v})$ formaldehyde, stirring for $20 \mathrm{~h}$ at room temperature and finally adding an equal volume of $40 \%$ formaldehyde. After total removal of the solvent, the solid residue was recrystallized from a solution in water/acetone (10:90). Three end-products were formed by conjugating the tuberculostatic compounds to the PEG-PASP polymer chain, as follows: isoniazid (INH, 14), rifampin (RMP, 16) or hydroxymethylpyrazinamide $\left(\mathrm{PZACH}_{2} \mathrm{OH}, 11\right)$ was dissolved in $\mathrm{N}, \mathrm{N}$-dimethylformamide, (DMF) and the solution added to the aqueous solution of PEG-PASP together with the condensing agent, EDC (12). After $4 \mathrm{~h}$ stirring at $0^{\circ} \mathrm{C}$, more EDC was added and the reaction mixture stirred at room temperature for $24 \mathrm{~h}$. The resulting solution was dialyzed against $0.1 \mathrm{M}$ acetate buffer ( $\mathrm{pH}$ 4.5) in a membrane with a 1000dalton cut-off for $4 \mathrm{~h}$, then against distilled water for an equal time and finally lyophilized to a semi-solid. The conjugated polymers thus obtained were PEG-PASP-PZA (13), PEGPASP-INH (15) and PEG-PASP-INH-RMP (17).

\section{Bacterial Strains}

The following strains of Mycobacterium were used to assay the polymer prodrug derivatives: M. tuberculosis standards $\mathrm{H}_{37} \mathrm{Ra}$ (American Type Culture Collection - ATCC 25177) and $\mathrm{H}_{37} \mathrm{Rv}$ (ATCC 27294), denoted $\mathrm{Ra}$ and $\mathrm{Rv}$ respectively; $M$. avium (denoted $\mathrm{Ma}$ - ATCC 25291), M. kansasii (Mk - ATCC 12478), M. intracellulare (Mi - ATCC 13950) and $M$. malmoense (Mm - ATCC 29571).

\section{Determination of Minimum Inhibitory Concentration (MIC) $)^{14}$ - in vitro assay}

All the wells around the edge of a 96-well microplate were filled with $200 \mu \mathrm{L}$ sterile distilled water. Next, the wells B to $G$ of columns 3 to 9 , 10 and 11 received, respectively, 100, 150 and $200 \mu \mathrm{L}$ of mycobacterial liquid growth medium, $7 \mathrm{H}_{9}$. Stock solutions of $8.9 \mathrm{mg} / \mathrm{mL}$ of the three synthetic polymer derivatives, their respective standard drugs $\left(\mathrm{INH}^{15}, \mathrm{PZA}^{16}\right.$ and $\mathrm{RMP}^{17}$ ) and the prodrug carrier (PEG-PASP) were used to prepare dilutions of 3,560 or $356 \mu \mathrm{g} / \mathrm{mL}, 50$ and $100 \mu \mathrm{L}$ of which were pipetted into wells $B$ to $G$ of columns 2 and 3 , respectively. After thorough mixing, $100 \mu \mathrm{L}$ of mixture was transferred from each well ( $B$ to $G$ ) in column 3 to the corresponding well in column 4 , then from column 4 to 5 and so on, until the final dilution in column 9, from which $100 \mu \mathrm{L}$ was discarded, after mixing. Lastly, $100 \mu \mathrm{L}$ of a suspension of mycobacteria was added to every well from $B$ to $\mathrm{G}$ in all columns except 2 and 11 . This inoculum was taken from a culture grown for 10 days in $7 \mathrm{H}_{9}$ medium at $37^{\circ} \mathrm{C}$ whose turbidity was adjusted to the McFarland No.1 scale and which was then diluted 25 times.

Thus, in the $M$. tuberculosis microplates, the diluted drug stock was $356 \mu \mathrm{g} / \mathrm{mL}$ and every well in columns 3 to 9 contained a final volume of 200 $\mu \mathrm{L}$, with drug dilutions between 89.0 and 5.56 $\mu \mathrm{g} / \mathrm{mL}$ and an inoculum of Ra or Rv bacteria. For the $\mathrm{Ma}, \mathrm{Mk}, \mathrm{Mi}$ and $\mathrm{Mm}$ assays, microplates were prepared with the $3560 \mu \mathrm{g} / \mathrm{mL}$ drug stocks, giving dilutions (in columns 3 to 9 ) of 890 to 55.6 $\mu \mathrm{g} / \mathrm{mL}$. The microplates were sealed with parafilm and incubated at $37^{\circ} \mathrm{C}$ for 5 days, after which $25 \mu \mathrm{L}$ of a freshly-prepared solution of the redox dye Alamar Blue (1:1 v/v) in 10\% Tween 


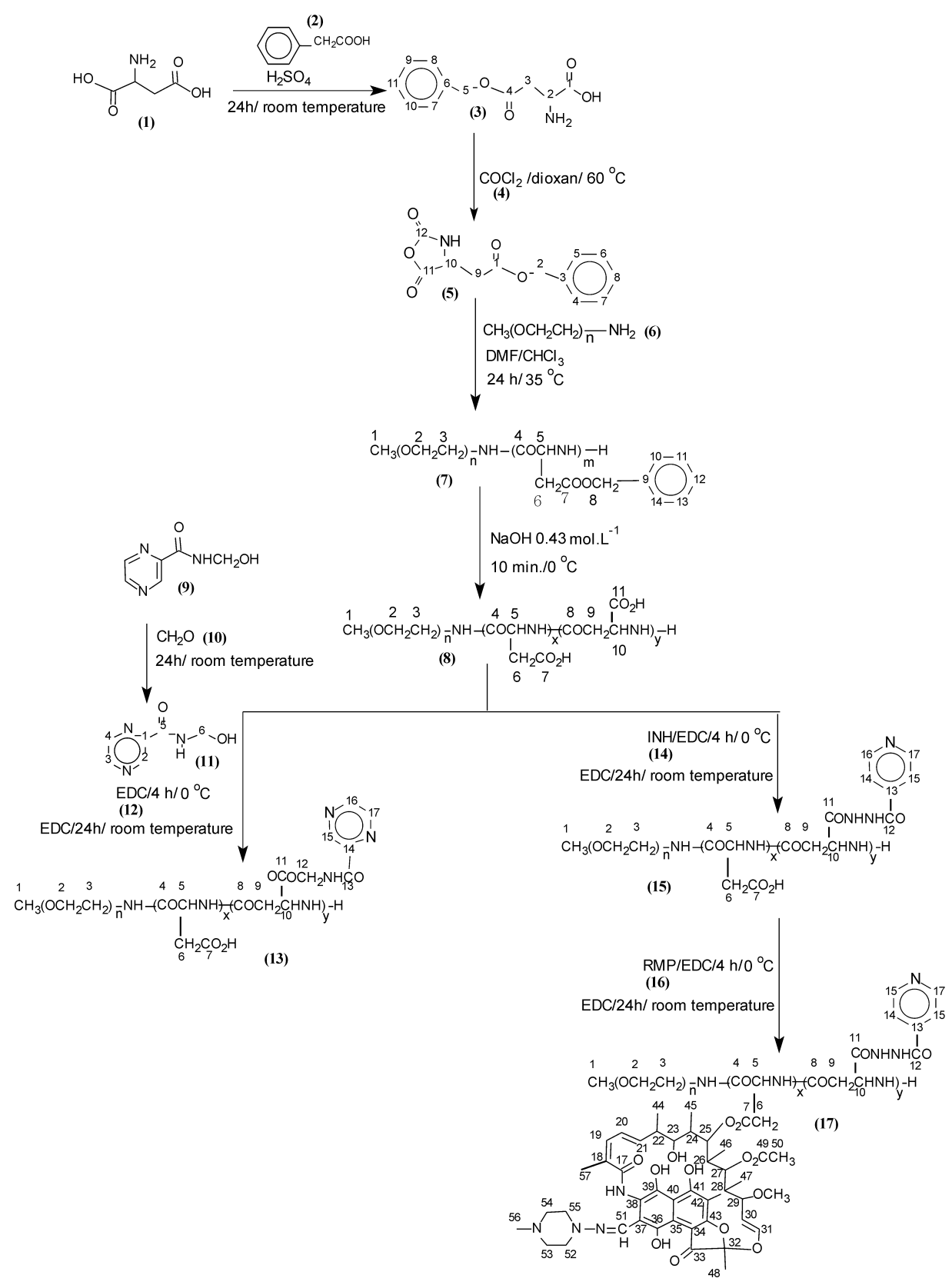

Scheme 1. Synthetic pathway for micelle-forming polymer derivatives of isoniazid, pyrazinamide and rifampin.

80 solution was added to well B10, which was resealed and the plate returned to the incubator for another 24h. Assuming the medium in well B10 turned pink (indicating cell growth), the Alamar Blue solution was added to all wells and the plate incubated at $37^{\circ} \mathrm{C}$ for another $24 \mathrm{~h}$, at the end of which the color of each well was recorded. The tuberculostatic MIC was taken as the lowest concentration of the drug that 
prevented the medium changing from blue to pink, i.e., which halted growth of mycobacteria.

\section{Determination of Critical Micelle Concentration (CMC)}

Aqueous solutions of PEG-PASP-PZA, PEGPASP-INH and PEG-PASP-INH-RMP were prepared, at concentrations ranging from $5.0 \times 10$ ${ }^{8}$ to $5.0 \times 10^{-1} \mathrm{mg} / \mathrm{L}$, and $10 \mathrm{mg}$ of the stain Sudan III was added to $10 \mathrm{~mL}$ to each solution. The absorbance of light by the solution at $519 \mathrm{~nm}$ was measured with a Shimadzu UV1601 PC spectrophotometer over a $1 \mathrm{~cm}$ light-path, to evaluate the apparent solubility of the stain in the micelle suspension. Each experiment was performed in triplicate.

\section{Estimation of Diameter of Micelles}

Solutions of $1 \%(\mathrm{w} / \mathrm{v}$ ) PEG-PASP-PZA, PEGPASP-INH and PEG-PASP-INH-RMP in water were passed through Amicon YM3 43mm PK ultrafiltration membranes. The diameters of the micelles in the filtered polymer solutions were then estimated by measuring their dynamic light scattering in a Malvern 4,700 MW system, equipped with a $60 \mathrm{~mW} \mathrm{He} / \mathrm{Ne}$ laser operating at $632.8 \mathrm{~nm}$ (Spectra-Physics 107) and a thermostatic bath. Each experiment was performed in triplicate.

\section{Determination of free carboxyl groups}

The proportions of free carboxyl groups in the derivatives PEG-PASP-PZA, PEG-PASP-INH and PEG-PASP-INH-RMP were found by titrating these polymers and the original copolymer PEG-PASP, in aqueous solution, against $0.01 \mathrm{M} \mathrm{NaOH}$ using phenolphthalein as indicator. Each experiment was performed in triplicate. Release of tuberculostatic drugs from the micelles Dialysis bags (cut-off 1000) each containing the INH, RMP and PZA-loaded micelle suspension with $300-400 \mathrm{mg} / \mathrm{ml}$ of $\mathrm{INH}$, RMP and PZA were placed in a dialysate with ten times excess volume at $37{ }^{\circ} \mathrm{C}$. Phosphate buffered saline (PBS; pH 7.4) or $0.1 \mathrm{M}$ acetate buffer ( $\mathrm{pH}$ 5.0) was used as the micelle medium and dialysate. At a certain time interval, the micelles solutions in the dialysis bag were sampled and subjected to photometric assay at $485 \mathrm{~nm}$ to determine the residual INH, RMP and PZA content in the micelle.

\section{Stability studies ${ }^{18}$}

For this purpose, lyophilized and aqueous solution of polymeric micelles were placed in a climatic chamber at $25 \pm 2{ }^{\circ} \mathrm{C}, 60 \%$ relative humidity for 3 months. Lyophilized forms were placed in $2 \mathrm{ml}$ Eppendorf tubes and solution forms were placed in $5 \mathrm{ml}$ colored bottle with a lid. Samples were taken to determine the drug content at the beginning and at the end of 3 months. Drug content was measured spectrophotometrically after extracting drug into toluene and UV spectra were also checked.

\section{RESULTS \\ Synthesis}

Derivatives of the copolymer PEG-PASP, containing moieties of the drugs pyrazinamide, isoniazid and rifampin, were successfully synthesized by condensation with free carboxyl groups on the PASP segments of the carrier. The drug substituents were satisfactorily detected in the purified polymer derivatives by spectrometric analysis and the non-polymer intermediates involved in the synthetic pathway were identified by elemental analysis, spectrometric analysis and spectrophotometry.

Taking the titrated free carboxyl groups in PEGPASP as $100 \%$, the proportion substituted by isoniazid was $65.02 \%\left(\equiv 4.03 \times 10^{-4} \mathrm{~mol} \mathrm{INH}\right.$ per gram PEG-PASP-INH). It follows that the fraction of free carboxyl groups in the INH derivative was $2.01 \times 10^{-4} \mathrm{~mol} \mathrm{COOH} / \mathrm{g}$. Out of these free groups, $52.5 \%$ were substituted by rifampin, giving a total of $1.05 \times 10^{-4} \mathrm{~mol}$ RMP per gram PEG-PASP-INH-RMP. Thus, the fraction of free carboxyl groups in PEG-PASP substituted by $\mathrm{INH}$ and RMP in the two-drug derivative was $82.04 \%$. In the pyrazinamide derivative, $85.7 \%$ $\left(4.9706 \times 10^{-4} \mathrm{~mol} / \mathrm{g}\right)$ of the free carboxyls were substituted by PZA. 


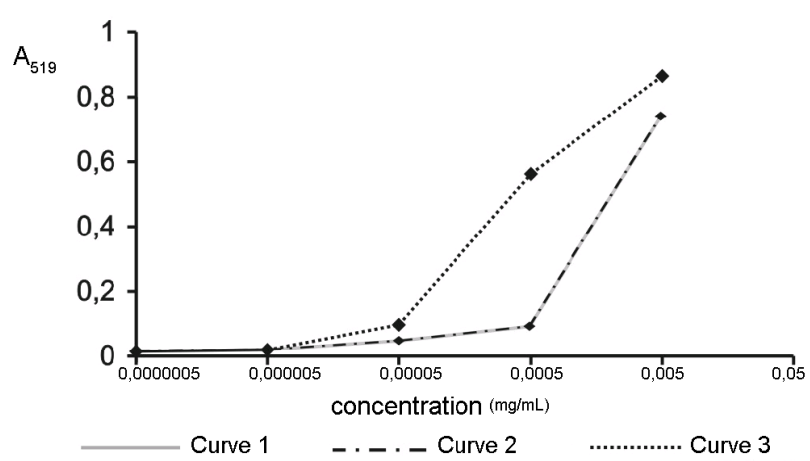

Fig 1: Determination of CMC of PEG-PASP-PZA (curve 1), PEG-PASP-INH (curve 2) and PEG-PASP-INH-RMP (curve 3).

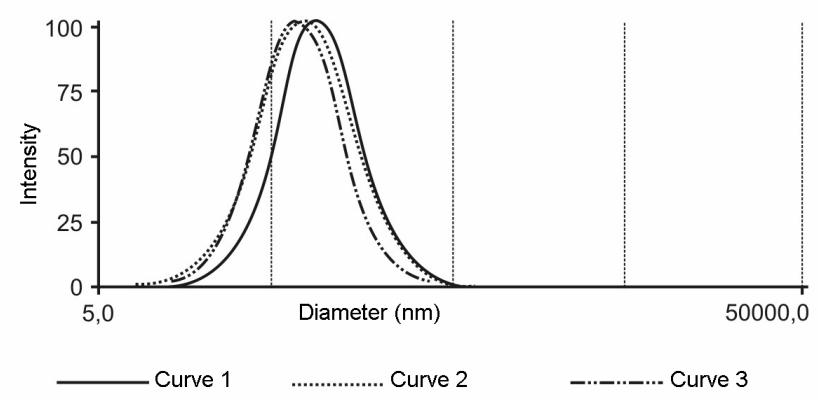

Fig 2: Determination of micelle diameter of PEG-PASP-PZA (curve 1), PEG-PASP-INH (curve 2) and PEG-PASP-INH-RMP (curve 3).

Table 1: MIC results for INH, RMP and PZA and their polymer derivatives

\begin{tabular}{|c|c|c|c|c|c|c|c|}
\hline Bacterial & & & & $\mathrm{MIC}(\mu$ & & & \\
\hline & INH & (15) & RMP & (17) & PZA & (13) & (PEG-PASP) \\
\hline $\mathrm{Ra}$ & $\leq 0.031$ & $\leq 0.031$ & $\leq 0.125$ & $\leq 5.56$ & $\leq 6.25$ & $\leq 6.25$ & I \\
\hline Rv & $\leq 0.031$ & $\leq 0.031$ & $\leq 0.125$ & $\leq 5.56$ & 12.5 & 12.5 & I \\
\hline Ma & $>128.0$ & $>128.0$ & $\leq 8.0$ & 111.2 & 162 & 162 & I \\
\hline Mk & $>128.0$ & $>128.0$ & $\leq 8.0$ & $\leq 55.6$ & 162 & 162 & I \\
\hline $\mathrm{Mi}$ & $>128.0$ & $>128.0$ & $\leq 8.0$ & $\leq 55.6$ & 162 & 162 & I \\
\hline $\mathrm{Mm}$ & 128.0 & $>128.0$ & $\leq 8.0$ & 445.0 & 162 & 162 & I \\
\hline
\end{tabular}

PEG-PASP - in vitro is devoid of pharmacological activity; (I: INATIVE)

(15): PEG-PASP-INH; (13): PEG-PASP-PZA; (17): PEG-PASP-INH-RMP 


\section{Critical Micelle Concentration}

To verify and characterize the formation of micelles, the CMC was determined by plotting the solubility of Sudan III stain (at an absorbance of $519 \mathrm{~nm}$ ) in aqueous solutions of the polymer derivatives against polymer concentration and observing the threshold above which micelles form, providing a phase in which the stain is soluble. The CMC of PEG-PASP-PZA and PEGPASP-INH was $5.0 \times 10^{-4} \mathrm{mg} / \mathrm{L}$ and that of PEGPASP-INH-RMP, $5.0 \times 10^{-5} \mathrm{mg} / \mathrm{L}$, as can be verified from Figure 1.

\section{Micelle Diameter}

The dynamic light-scattering results displayed in Figure 2 shows that the mean diameters of the micelles were: $78.2 \mathrm{~nm}$ for PEG-PASP-PZA, $84.2 \mathrm{~nm}$ for PEG-PASP-INH and $98.9 \mathrm{~nm}$ for PEG-PASP-INH-RMP.

\section{Antimycobacterial activity}

The antibacterial MIC results (in a $\mathrm{pH} 7$ medium) are expressed in terms of the total weight of each derivative or drug standard. INH, RMP, PZA and their derivatives were tested against the mycobacterial strains ( $\mathrm{Ra}, \mathrm{Rv}, \mathrm{Ma}, \mathrm{Mk}, \mathrm{Mi}$ and $\mathrm{Mm}$ ). In a preliminary assay, $\mathrm{PZACH} \mathrm{CH}_{2} \mathrm{OH}$ was tested against $\mathrm{Ra}$ and exhibited tuberculostatic activity (MIC of $24.8 \mu \mathrm{g} / \mathrm{mL}$ ). The polymer derivatives showed tuberculostatic activity and the MICs observed are displayed in Table 1.

\section{Stability studies}

Stability test of micelles consisted of visual control and analytical measurement of drugs contents.

No sedimentation or phase separation was observed during the 3-month stability test. The spectrophotometric spectra remained unchanged after 3 months, showing that no chemical degradation took place. Lyophilized of polymeric micelles did not change in 3 months showing the high storage stability of this formulation. In the aqueous form of polymeric micelles, a 12,33\%, $14,21 \%$ and $16,32 \%$ decrease in INH, PZA and RMP, respectively.

\section{DISCUSSION}

Our approach to tuberculostatic delivery through polymeric micelles was to design with care the chemical composition and architecture of the amphiphilic polymers forming the polymeric micelle. By the condensation of hydroxymethylpyrazinamide, isoniazid and rifampin with free carboxyl groups on the copolymer poly(ethyleneglycol)-poly(aspartic acid), micelle-forming carrier-drug conjugates were obtained. The presence of a covalently bonded drug molecule may have a steric effect on the core packing that could potentially impact the release rate of the drug. In addition, covalently linked tuberculostatic agents will be released when the ester or amide linkages are cleaved in the same manner as the polymer backbone. This has been shown in other systems to extend release over physically entrapped drug.

When the concentration reaches the CMC, the physicochemical properties of solutions of amphiphilic molecules go through an abrupt change. Therefore, the CMC can be found by observing the variation, with concentration, of properties such as hydrophobic dye solubility, surface tension or conductivity. While the CMC is not really a single concentration but a band, its range is often found to be sufficiently narrow for the band to be treated as a point ${ }^{20,21}$. The CMC of polymeric micelles can be estimated by fluorescence spectroscopy. The CMCs of PEGPASP-PZA, PEG-PASP-INH and PEG-PASPINH-RMP were obtained from curves representing the process of solubilization of Sudan III in their solutions, at various polymer concentrations in the range $5 \times 10^{-8}$ to $5 \times 10^{-1}$ $\mathrm{mg} / \mathrm{L}$. The onset of micelle formation can be seen clearly, in plots of light absorbance against concentration, as a sharp change in the slope of the curve, which occurs where the dye, insoluble in water but soluble in the hydrophobic compartment in the micelle, starts to be taken up by the solution. Thus, below the CMC, the curve is virtually a plateau, while above this concentration it rises steadily with the number of micelles formed. In this way, the CMC was found to be $5.0 \times 10^{-4} \mathrm{mg} / \mathrm{L}$ for PEG-PASP-PZA and PEG-PASP-INH and $5.0 \times 10^{-5} \mathrm{mg} / \mathrm{L}$ for PEGPASP-INH-RMP. 
The size of micelles is controlled by the length of the core-forming segment and the length of the corona-forming chain ${ }^{18,19,21}$, but it depends also on the method of micellization selected for the preparation of micelles. For micelles produced using the dialysis method, it varies depending on the organic solvent employed. The choice of organic solvent also affects the yield of micellization. Note that, above all, it is important to select conditions that minimize the formation of large polymer aggregates (diameter $>300$ $\mathrm{nm}$ ), in addition to the desired polymeric micelles ${ }^{7,21}$.

The mean micelle diameters of PEG-PASP-PZA, PEG-PASP-INH and PEG-PASP-INH-RMP were shown, by the technique of dynamic light scattering, to be 78.2, 84.2 and $98.9 \mathrm{~nm}$, respectively, and the distribution of diameters has a single peak, as can be seen in the curves. In all cases, the size distribution was unimodal, indicative of the absence of free polymer chains and of large aggregates. In order to obtain such small micelles, with a monomodal size distribution, several precautions were taken during the preparation of the derivatives: rigorous control of temperature and constant stirring during the synthesis of PEG-PBLA, followed by sonication of the product mixture for half an hour; elution of the derivative solutions through a Sephadex® column, with distilled water, and ultrafiltration through a YM3 $43 \mathrm{~mm}$ 10 PK membrane. It has been shown that the uptake of particles within the intestine and the extent of drug absorption increase with decreasing particle size and increasing specific surface area ${ }^{21}$. Thus, the small size exhibited by all the polymeric micelles studied shows a favorable trend toward oral drug delivery. Since polymeric micelles are a relatively new carrier, it is important that several system characteristics be elucidated ${ }^{22,23,24}$, such as stability, mechanism release and activity.

Micellar structure is strongly affected by temperature. The temperature at which micelles are formed is known as critical micellization temperature (CMT). For most copolymers this value is $25-40{ }^{\circ} \mathrm{C}$. If micelles were kept in refrigerator, temperature would fall lower than
CMT, thus micelles would lose their intact structure and drug would precipitate. For this reason, stability test was performed at $25^{\circ} \mathrm{C}$.

The present study corroborates the view of Yokoyama et $a^{\beta}$ and $\mathrm{Hans}^{20}$ since the drugs incorporated there - isoniazid, pyrazinamide and rifampin - exhibit a rather strict structure-function relationship that would demand release of the drug moiety for the biological activity to be effective, i.e. the micelles act as prodrugs.

There is an increasing appreciation for the role of nontuberculous mycobacteria as pathogens causing pulmonary disease, disseminated disease, or both in immunocompetent and immunocompromised individuals ${ }^{25,26,27,28}$. Mycobacterium kansasii usually causes chronic lung infection resembling pulmonary tuberculosis. Cases of soft tissue or bone and joint infections have also reported. The recommended treatment regimen for these infections is a combination of rifampin, isoniazid, and ethambutol ${ }^{25,26,27}$. M. xenopi, M. simae, and $M$. malmoense are infrequent human pathogens but also appear to be increasing in prevalence ${ }^{27}$. Particularly $M$. malmoense next to $M$. tuberculosis and $M$. avium complex, it is the most common cause of mycobacterial infection in certain geographic locations ${ }^{26,29}$. These nontuberculous mycobacteria species cause lymphadenitis or chronic pulmonary infection. The in vitro susceptibilities of these organisms and the results of chemotherapy have been variable. Conventional therapy of $M$. malmoense infections with antituberculosis drugs is often of limited value, and there is thus a need for improved drug regimens ${ }^{27}$. Like most other atypical mycobacteria, it has a high degree of resistance to antibacterial drugs, including specific antituberculosis drugs such as $p$ aminosalicylic acid, ethambutol, isoniazid and pyrazinamide.

As mentioned in the experimental section, PZA, $\mathrm{INH}, \mathrm{RMP}$ and polymer derivative were tested against seven strains of Mycobacterium. When the MICs of the polymer derivatives were recalculated in terms of the weights of the active drug moieties conjugated to the PEG-PASP carrier, instead of the total weight of polymer, the 
increase in activity (decrease in MIC) of the polymer-bound drugs, relative to the free, was about 15-fold for PEG-PASP-INH and PEGPASP-INH-RMP and about 13-fold for PEGPASP-PZA. In this sense, the micelle-forming derivatives all showed stronger antimycobacterial activity than the original drugs. Of particular note, good activity was obtained against strains considered drug-resistant and commonly recovered from HIV-infected patients. This potentiation of the drug activity when conjugated to the polymer is clearly promising with regard to possible reductions in the dose and, hence, in the appreciable toxicity of these drugs.

\section{CONCLUSION}

Polymeric micelles of tuberculostatics were obtained, characterized and tested against Mycobacterium strains, showing excellent characteristics, as promising drug carrier systems. In the organism expected that the drugs are released following internalization of micelle, by cleavage, i.e. its act as prodrugs These micelles are able to increase a hydrophobic drug's solubility, to reduce drug toxicity, improve drug bioavailability, target drug delivery to a specific site, and sustain release systemically, prolonging the drug action.

\section{ACKNOWLEDGEMENT}

The authors are grateful to FURP (São Paulo, Brazil) for donating the tuberculostatic drugs; to FAPESP (Fundação de Amparo à Pesquisa do Estado de São Paulo, São Paulo, Brazil) for financing the work; and Tim Roberts for useful suggestions and help with the English text.

\section{REFERENCES}

1. World Health Organization; Global tuberculosis control: WHO Report 2006 surveillance, planning, financing; Geneva: Available in the Internet. http://www. who.int/tb/publications/global report/20 06/pdf/full report.pdf , June, 2007.

2. World Health Organization. Tuberculosis control and research strategies for 1990s: Memorandum from WHO meeting. Bull WHO 1992, 70, 17.

3. Wermuth MCG. Strategies in the search for new lead compounds or original working hypotheses. In: Wermuth, M.C.G. The practice of medicinal chemistry; New York, Academic Press, 1996.

4. Testa B. Prodrug research: futile or fertile? Biochem Pharmacol. 2004; 68:2097.
5. Yokoyama M, Inoue S, Kataoka, K, Yui N, Sakurai Y. Preparation of adriamycin-conjugated poly(ethylene glycol)-poly(aspartic acid) block copolymer. A new type of polymeric anticancer agent. Makromol. Chem. Rapid. Commun. 1987; 8: 431.

6. Yokoyama M, Miyauchi M, Yamada N, Okano T, Sakurai Y, Kataoka K. Inoue, S. Polymer micelles as novel drug carrier: adriamycin-conjugated poly(ethylene glycol)-poly(aspartic acid) block copolymer. In: ANDERSON, J.M.; KIM, S.W.; KNUTSON, K. (Ed). Advances in drug delivery systems; Elsevier, Amsterdam, 1987.

7. Cammas S, Kataoka K. Functional poly(ethylene oxide)co-( $\beta$-benzyl-L-aspartate) polymeric micelles: block copolymer synthesis and micelles formation. Makromol. Chem. Phys. 1995; 196:1899.

8. Kataoka K, Harada A, Nagasaki Y. Block copolymer micelles for drug delivery: design, characterization and biological significance Adv Drug Deliv Rev. 2001; 47:113.

9. Silva M, Lara AS, Leite CQF, Ferreira E.I. Potential tuberculostatic agents: micelle-forming copolymer poly(ethylene glycol)-poly(aspartic acid)prodrug with isoniazid Arch. Pharm. 2001; 334:189.

10. Silva $M$, Ricelli NL, Valentim CS, Ferreira AG, Sato $D$, Leite CQF, Ferreira El. Potential tuberculostatic agent: micelle-forming pyrazinamide prodrug. Arch. Pharm.. 2006; 339: 283.

11. Benoiton LA. Synthesis of isoasparagine from $\beta$-benzyl aspartate. Can. J. Chem. 1962; 40:570.

12. Fuller WD, Verlander MS, Goodman M. A procedure for the facile synthesis of amino acid $\mathrm{N}$ carboxyanhydrides. Biopolymers 1976; 15: 1869.

13. Mobashery S, Jonhston M. A new approach to the preparation of $N$-carboxy $\alpha$-amino acid anhydrides. J. Org. Chem. 1985; 50: 2200.

14. Franzblau SG, Witzig RS, Mclaughlin JC, Torres $P$, Madico G, Hernandez A, Degman MT, Cook MB, Quenzer VK, Ferguson RM, Gilmar RH. Rapid, low-technology MIC determination with clinical Mycrobacterium tuberculosis isolates by using the Microplate Alamar Blue Assay. J. Clin. Microb. 1998; 32: 362.

15. Brewer GA. Isoniazid. Anal. Profiles Drugs Subst. 1977; 6:186.

16. Felder E, Pitre D. Pyrazinamide. Anal. Profiles Drugs Subst. 1983; 12: 433.

17. Radalli P, GalloGG.. Rifampin. Anal. Profiles Drugs Subst. 1985; 12:467.

18. Sezgin Z, Ksel NY, Baykara T. Preparation and characterization of polymeric micelles for solubilization of poorly soluble anticancer drugs Eur. J. Pharm. Biopharm. 2006, 64:261-268

19. Kataoka $K$, Matsumoto $T$, Yokoyama M, Okano $T$, Fukushima S, Okamoto K, Kwon, GS. Doxorubicinloaded poly(ethylene glycol)-poly(b-benzylLaspartate) copolymer micelles: their pharmaceutical characteristics and biological significance. J. Control Release. 2000; 64:143153 


\section{Márcia et al}

20. Hans ML. Synthesis, characterization, and application of biodegradable polymeric prodrug micelles for longterm drug delivery. Thesis. Faculty of Drexel University, 2005

21. Francis M.F, Cristea M, Winnik FM. Polymeric micelles for oral drug delivery: Why and how Pure Appl. Chem., 2004; 76:1321-1335.

22. Attwood D, Florence AT. Surfactants systems: their chemistry, pharmacy and biology; New York: Chapman and Hill, 1983, pp 72-117.]

23. Flory PJ. Molecular organization in micelles and vesicles. Proc. Natl. Acad. Sci. 1981; 78: 676.

24 Lindman B, Wennerstrom H, Gustavsson H, Kamenka N, Brun B. Some aspects on the hydration of surfactant micelles. Pure Appl. Chem. 1980; 52: 1307.
25. Henry, M.T., Inamdar, L. O'Riordain, D., Schweiger, M., Watson, J.P. Nontuberculous mycobacteria in nonHIV patients: epidemiology, treatment and response. Eur Respir J 2004; 23:741-746

26. Ornedrod, P. A step forward in the evidence-based treatment of opportunist mycobacteria Thorax 2001; 56:163

27. Klemens SP, Cynamon MH. Activities of azithromycin and clarithromycin against nontuberculous mycobacteria in beige mice. Antimicrob. Agents Chemother., 1994; 38:1455-1459.

28. Kourbeti IS, Maslow MJ. Nontuberculous mycobacterial infections of the lung.Curr Infect Dis Rep. 2000; 2:193-200.

29. Hoffner SE, Hjelm U, Kallenius G. Susceptibility of Mycobacterium malmoense to Antibacterial Drugs and Drug Combinations. Antimicrob. Agents Chemother., 1993; 37:1285-1288 